\title{
Changing the Impact of Nursing Assistants' Education in Seniors' Care: the Living Classroom in Long-Term Care
}

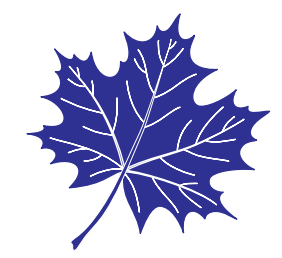

Veronique M. Boscart, RN, $\mathrm{PhD}^{1,2,3}$, Josie d'Avernas, $\mathrm{MSc}^{2}$, Paul Brown ${ }^{3}$, Marlene Raasok, BSCP, MHSA ${ }^{2}$

${ }^{I}$ Conestoga College Institute of Technology \& Advanced Learning, Kitchener, ON; ${ }^{2}$ Schlegel-University of Waterloo Research Institute for Aging, Waterloo, ON; ${ }^{3}$ Schlegel Villages, Kitchener, ON

DOI:https://doi.org/10.5770/cgj.20.238

\section{ABSTRACT}

\section{Background}

Evidence-informed care to support seniors is based on strong knowledge and skills of nursing assistants (NAs). Currently, there are insufficient NAs in the workforce, and new graduates are not always attracted to nursing home $(\mathrm{NH})$ sectors because of limited exposure and lack of confidence. Innovative collaborative approaches are required to prepare NAs to care for seniors.

\section{Methods}

A 2009 collaboration between a NH group and a community college resulted in the Living Classroom (LC), a collaborative approach to integrated learning where NA students, college faculty, $\mathrm{NH}$ teams, residents, and families engage in a culture of learning. This approach situates the learner within the $\mathrm{NH}$ where knowledge, team dynamics, relationships, behaviours, and inter-professional (IP) practice are modelled.

\section{Results}

As of today, over 300 NA students have successfully completed this program. NA students indicate high satisfaction with the $\mathrm{LC}$ and have an increased intention to seek employment in NHs. Faculty, NH teams, residents, and families have increased positive beliefs towards educating students in a NH.

\section{Conclusion}

The LC is an effective learning approach with a positive and high impact learning experience for all. The LC is instrumental in contributing to a capable workforce caring for seniors.

Key words: gerontology, education, nursing home, long-term care, residents, seniors, nursing assistants, integrated learning

\section{INTRODUCTION}

The proportions of seniors are steadily increasing. ${ }^{(1)}$ On July 1, 2015, approximately one in six Canadians (16.1\% or $5,780,900$ persons) was at least 65 years old, and according to population projections, the number of persons aged 65 years and older is expected to continually increase and account for $20.1 \%$ of the population by July 1,2024 . $^{(2)}$ However, seniors over the age of 65 are likely to have one or more chronic illness, ${ }^{(3)}$ which can compromise their prospect of independence and indicate the need for health-care professionals (HCPs) capable of addressing these needs in a variety of care settings.

The largest proportion of HCPs providing care and services to seniors are unregulated staff (a.k.a. Nursing Aides or Assistants [NAs], Resident Care Attendants or Personal Support Workers). These HCPs are faced with the rising challenge of caring for an increasingly complex group of seniors with diverse biomedical and psychosocial needs. Yet, gerontological education is not a priority in most health-care programs. ${ }^{(4)}$ The integration of gerontological courses into health-care programs is scarce and, although seniors have more contacts with HCPs, there is a lack of HCPs with geriatric expertise. ${ }^{(5)}$ Furthermore, competencies offered in health-care programs often lack clearly expressed standards for gerontology practice. ${ }^{(6)}$ Present gaps in HCP competencies hinder the necessary efforts to build and attract a well-equipped workforce in seniors care, resulting in recruitment and retention challenges. Thus, there is a need for improved and innovative programs to accommodate the demand for gerontological expertise among health-care students.

This manuscript presents the Living Classroom (LC), an innovative pedagogical approach, collaboratively created by Conestoga College and Schlegel Villages, a continuum of seniors care in Ontario, Canada. The LC presents an inter-professional (IP) learning approach whereby a post-secondary educator (PSE) program is delivered within the context of a Nursing Home $(\mathrm{NH})$, with team members consisting of faculty, students, NH teams, residents, and families who engage with each other within a culture of interactive learning. 


\section{NA Education}

In one Ontario college, the NA program provides students with a thorough understanding of person-centred are delivery in a variety of settings. Students progress from novice engagement to a professional level so that, upon graduation, they are competent and confident to participate in care as directed. NA students receive theoretical learning (lectures), as well as applied and experiential learning (skills lab, simulated environments, and clinical placements). The transfer of knowledge from a theoretical concept to an applied skill or behaviour is often difficult for students, ${ }^{(4)}$ but essential if one wants to successfully graduate and practise. This knowledge transfer is particularly challenging in gerontology, as seniors often present with complex care needs requiring comprehensive care.

Baumbusch and Andrusyszyn ${ }^{(7)}$ state that students must first acquire gerontological knowledge in the classroom, so that they may then develop skills specific to the care for seniors. However, of equal importance is the clinical aspect of learning, as it has been found that students are more likely to apply their theoretical learning to care of seniors if they work with clinical experts in the field. It is clear that both didactic and clinical components of learning have their place in gerontological education. ${ }^{(4)}$

When these learnings occur independently, disconnects happen between education and practice. For example, NA students are taught to transfer a senior from a bed to a chair in a theoretical manner, followed by practice sessions with fellow students. Once students can perform this skill adequately, learning is moved to a practice setting, in which students are expected to integrate theoretical learning into a personal care encounter. This situation is very different from the theoretical or lab learning setting, as the student is now expected to help a senior with a transfer, yet the senior presents with real mobility impairments and potential other frailties. This situation requires students to not only perform the 'transfer', but also maintain a person-centred conversation, observe mobility and other capabilities, and determine and adjust the care plan based on these observations. It is not possible to fully develop these essential skills in a classroom or even a controlled lab setting with healthy participants; this independent learning leads to a theory-practice divide. The Council of Ontario Universities has termed this disconnect of didactic and clinical education as a "crisis" in education of HCPs. ${ }^{(8)}$

In order to maximize the knowledge transfer, both didactic and clinical components need to be integrated into an immersive form of learning as opposed to keeping these as separate forms of knowledge acquisition. In turn, this integrated learning enhances the development of a strong workforce and drives quality care. ${ }^{(8)}$

\section{Teaching Long-Term Care Homes}

Successful gerontological learning in actual care settings has been described by several authors. In the 1980s, a model for the teaching nursing home (TNH) was developed and found to be effective in improving care for NH residents by supporting nursing students' clinical education in that setting. (9) The researchers described a partnership model developed between a nursing college and four NHs. Positive outcomes included increased professionalism for the NH team, identification of areas for growth within the NHs, increased awareness of how the NH team's practice influenced new nurses, and increased interest among students in choosing a $\mathrm{NH}$ environment as a career. ${ }^{(9)}$

Wieland and colleagues ${ }^{(10)}$ described an academic $\mathrm{NH}$ program designed to improve care of residents, provide IP training for a medical team and allied health students, and stimulate research. Students were paired with an expert within their respective field to integrate what they learned in the classroom. Benefits of the program included significant decreases in resident transfers to acute care hospitals, as well as noteworthy improvements in residents' functional status, satisfaction, and morale.

Lastly, Mezey and Lynaugh ${ }^{(11)}$ created a five-year TNH program to improve quality of $\mathrm{NH}$ care, create a supportive environment for undergraduate and graduate nursing students and care teams, and promote clinical research. Funding ended in 1987; by then the program included 12 NHs and 11 university schools of nursing. The program resulted in 1.5 to 2 new full-time positions in each $\mathrm{NH}$ - primarily nurse practitioners and clinicians specializing in geriatrics - and these positions focused on education, quality assurance and infection control. (12) The researchers' analysis of the TNH program revealed many benefits: resident hospitalization dropped 7\% in the $\mathrm{TNH}$ while rising 5\% in homes not linked to schools, and overall quality of care increased. Furthermore, the program cost was neutral; the cost of hiring of practitioners/clinicians was offset by reduced resident hospitalization. ${ }^{(12)}$

Although shown to be effective, the idea of the TNH faded in the 1990s because of a lack of funding. The concept has recently re-emerged in Canada as a viable approach, with a stronger emphasis on creating work-integrated learning opportunities for students to improve recruitment and retention of graduates in geriatric care settings. ${ }^{(9)}$

\section{METHODS}

\section{The Living Classroom}

In 2009, Conestoga College and Schlegel Villages embarked on a partnership to develop a pedagogical approach for contextual based learning for nursing and NA students in the context of a NH. Conestoga College is a community college in Ontario, Canada that offers polytechnic education "through a full range of career-focused education and applied research programs to prepare students for success". (13) Schlegel Villages is an owner and operator of NHs and retirement villages across Ontario, Canada, with the mission, "to provide holistic health care in a home environment, 
with emphasis on optimal health and life purpose for each resident". (13) Together, they developed the Living Classroom (LC). We developed a LC conceptual framework, where NA students, college faculty, and NH staff, residents, and families engage in the implementation of a culture of learning and mentoring within the physical context of a NH. This conceptual framework is unique, as it situates the learner within an environment where new knowledge, culture, relationships, behaviours, and practices are modelled, as opposed to exposing the learner to these concepts later on in the curricular program. Students are immersed for the entirety of their program in the actual milieu in which they will practice.

The LC model presents with three objectives. First, the LC aims to demonstrate strong partnerships between a post-secondary educator, an $\mathrm{NH}$, and the community, to develop meaningful and positive change in the education of HCPs who will provide care to seniors and their families. Second, the LC aims to create welcoming environments embracing interaction and mentoring among students, college faculty, $\mathrm{NH}$ staff, residents, and families to build and strengthen a culture of learning. The final aim of the LC is to integrate students into an environment where they learn and reflect on the life of seniors residing in NHs.

\section{A Framework for a Successful LC}

Over the last six years, the LC has been successful in meeting its objectives. To date, 312 students have graduated. A second LC opened in September 2015, welcoming another 96 students. Over the years, the collaborative has refined its conceptual framework and is now confident that others can benefit from this knowledge. The framework consists of four layers: exploring the potential for the $\mathrm{LC}$, developing the foundations for the $\mathrm{LC}$, implementing the $\mathrm{LC}$, and promoting and sustaining the LC. The layers are represented in 10 building blocks, discussed in further detail in Figure 1.

\section{Exploring the Potential for the LC}

Exploring the potential for the LC forms the basis for all the layers that follow. It encompasses three core building blocks: Agreeing on the LC definition, determining operational viability, and identifying win-win opportunities and developing shared values. Agreeing on a LC definition describes both partners engaging in the development of a clear conceptual definition to promote a shared understanding of the LC. This building block aims to promote realization that a $\mathrm{LC}$ is more than a classroom located within a $\mathrm{NH}$; it is an approach to integrated learning through experiences, a culture of learning, and supportive leadership. The second building block determines the operational viability and includes an assessment of the legal underpinnings for the presence of a post-secondary educator in the $\mathrm{NH}$, market viability (enrolment capacity, workforce needs), operational viability (scope

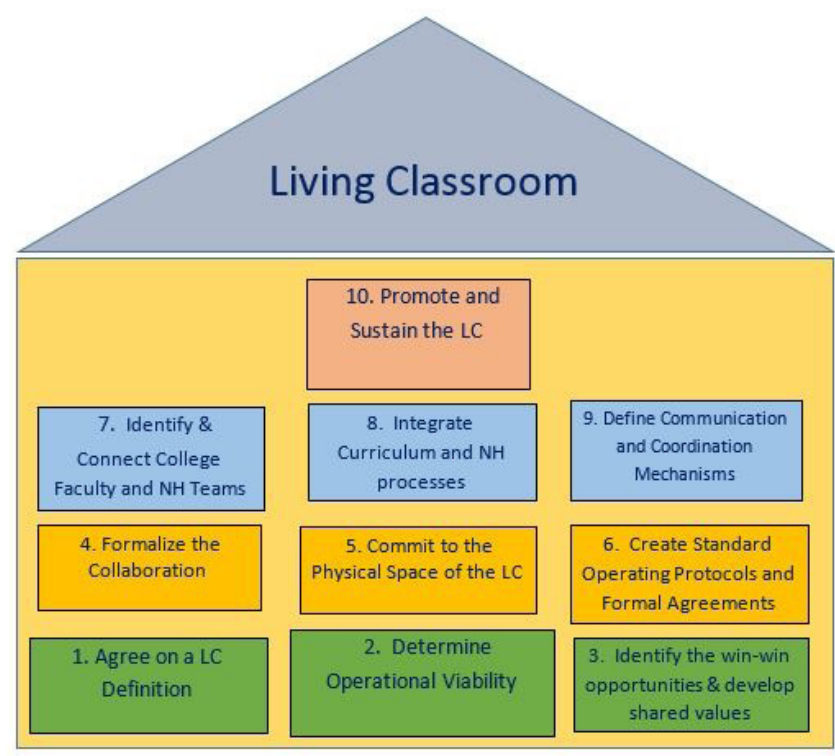

FIGURE 1. The building blocks of the Living Classroom

of education programs and human resource implications), and physical resources and financial viability (clarity on fiduciary relationships, space agreements outlining insurance and risk management considerations, and documented financial expectations). The third building block relates to identifying win-win opportunities and developing shared values, and describes the process of identifying successful collaborators and exploring shared objectives for the LC (learning from role models, NH staff acts as mentors), the shared reality of a LC (local access to education, recruitment pool for $\mathrm{NH}$ ), and direct benefits of the LC (increased support for NH learning, opportunities to expand access to clinical sites).

\section{Developing the Foundations for an LC}

The second level of the LC framework encompasses the foundational requirements in three building blocks: formalizing a collaboration, committing to the physical space of the LC, and creating standard operating protocols and formal agreements. Forming a collaboration is situated in a business relationship model. The business structure for a LC encompasses a shared vision and team work. Resources are shared, making the collaboration greater than the sum of its parts. The collaboration requires a high level of mutual trust and strong communication channels between the collaborators and internal and external stakeholders. The next building block describes the commitment to the physical space of the LC. This discussion refers to the locations and spaces available (labs, classrooms, washrooms), as well as the esthetics of the environment, the responsibility for care equipment and teaching technology, utilities, housekeeping and maintenance, faculty and student space and supports, safety, parking, and signage. Lastly, the foundation for the LC involves standard operating protocols and formal agreements. Operating procedures were developed 
prior to the LC opening, and required refinement once the LC was operational. Examples include procedures related to mandatory vaccinations for HCP students at the beginning of the LC (as opposed to the start of the first clinical placement for other students) or the distinction between students attending the $\mathrm{NH}$ as a volunteer or as a student. As well, it was determined that some existing operating procedures, both for the $\mathrm{NH}$ (introducing the LC to new residents) and for the educator (hand hygiene practices), required revisions to include both perspectives. As for the formal agreements, two agreements are recommended to protect the interests of the collaborative: a Use of Space Agreement and a Clinical Affiliation Agreement.

\section{Implementing an $\mathrm{LC}$}

Building on the previous layers, this third layer focuses on implementing an LC, and consists of three building blocks: identifying and connecting college faculty and $\mathrm{NH}$ teams, integrating curriculum and $\mathrm{NH}$ processes, and defining communication and coordination mechanisms. The first building block describes the importance of identifying and connecting college faculty and NH teams. Successful teaching and learning teams require both the faculty and the $\mathrm{NH}$ team to be dedicated to the LC. All members on the team must understand their individual roles, while maintaining an understanding of the values and objectives of the LC. Both faculty and members of the NH team develop strong relationships with all stakeholders and exemplify the values of student-centred learning for person-centred care. Providing consistent staffing by the college and the NH increases success.

The second block describes the intentional integration of the curriculum and $\mathrm{NH}$ processes. A curriculum is the heart of any given educational program and consists of a series of planned instruction that is coordinated in a manner that is designed to result in students' achievement of specific knowledge and skills and the application of knowledge. ${ }^{(14)}$ In the LC, additional integrated experiences are added to promote the experiential learning within a NH setting.

The third and last building block in this layer describes the communication and coordination mechanisms to implement the LC. This building block refers to the process of informing all stakeholders on a regular basis, to ensure that information is exchanged to help organize, implement, and evaluate all processes, resources, and services to sustain the LC. Of special importance are the resident and family communication mechanisms to highlight the significance of their engagement in the LC. Residents and their families are crucial partners in the creation of positive learning environments, and have extensive contributions to the students' understanding of person-centred care and a positive culture in aging.

\section{Promoting and Sustaining the LC}

The final level of the framework discusses the evaluation and strategies for promotion and sustainability of the LC.
This level consists of promoting the LC, developing strong connections with community partners, and monitoring of success. Promoting the LC is an ongoing strategy to create sustainability for the initiative. Once the LC is operational, the focus changes to developing strategies to sustain momentum, improve quality, and promote continuous innovation. This requires both organizations to work together to intentionally develop new possibilities for advancing the LC initiative in ways that are mutually beneficial. Part of this work lies in the forming of community connections in a variety of ways. To be successful, the LC must be recognized as a community resource, and specific attention must be given to the breadth of student learning and career preparedness, $\mathrm{NH}$ team development, and resident and family engagement. Lastly, one ought to monitor the LC success. Several strategies are used to represent the impact of the LC on people and systems, how this has been measured, and how the findings have been incorporated for further enhancement.

This framework described the four layers of exploring the potential for the LC, developing the foundations for the LC, implementing a LC, and promoting and sustaining the LC, represented by 10 building blocks. The partners' context, students, college faculty, $\mathrm{NH}$ teams, residents, and families all play vital roles in making the LC a success. Many teachable moments are created beyond knowledge and skill acquisition, including interpersonal communication and relationship building, team culture, workload management, IP relations and more.

\section{DISCUSSION}

The LC is an IP approach whereby a PSE program is delivered within the context of an $\mathrm{NH}$, with the team members consisting of faculty, students, NH teams, residents, and families, who engage with each other within a culture of interactive learning. Students are intentionally involved to develop their knowledge, behaviours, and skills in care for seniors and their families. This manuscript presents the conceptual framework and the operationalization for the LC designated for NA programs. The outcomes of the integration between learning and caregiving have been mutually beneficial, both for the college and the $\mathrm{NH}$, in that integration of knowledge transfer is established, promoted, and rewarded.

The paradigm behind the development of the first $\mathrm{LC}$ was to promote knowledge transfer within rich environments for work-integrated learning so students would be encouraged and well prepared for providing care to seniors. As noted by others, ${ }^{(11,12)}$ the idea of a NH is extremely valuable to promote knowledge transfer. Within an LC, students gain more and different knowledge, and demonstrate an increased comfort and confidence in working with residents, families, and teams. As well, students are exposed to a variety of learning opportunities and feel that their contributions are valued because they function as part of a care team while learning. This integrated approach of combining didactic and clinical 
components of learning has proven to be successful for a student's learning. ${ }^{(4,6)}$

College faculty are involved in the development of an appropriate LC curriculum, while respecting all standard program requirements for each of the programs. Faculty integrate the context and expertise of the NH teams, residents, and families into the learning with the aim to continually increase gerontological knowledge, confidence, and competence. Because of the NH context, faculty have the ability to integrate all aspects of learning; especially those situations that are difficult to simulate in a theoretical environment (i.e., a resident fall, a death of a resident, a quality audit), as well as having access to the highest expertise and the newest practices, regulations, and resources in the field. This observation has been made by previous researchers in the domain of teaching nursing homes. ${ }^{(11)}$

The NH team is presented with many opportunities to participate in mentoring of students and provides support and constructive feedback. Benefits for the NH team extend beyond active participation in developing the next workforce generation; because of the students' presence, the NH team is expected to present themselves as role models in relationships, IP collaborations, and evidence-informed care delivery. The phenomenon of increased quality of care because of mentorship roles has been discussed before, ${ }^{(9)}$ but is confirmed by the LC approach.

Residents and families contribute to teaching by sharing stories and experiences. The incorporation of meaningful activities for residents allows them to be part of something bigger, and encourages seniors to share wisdom. This engagement provides a sense of belonging which is greatly beneficial to NH residents, as discussed by Gilhooly and colleagues. ${ }^{(15)}$ Some researchers propose that meaningful activity leads to decreases in depression and anxiety for people with dementia $^{(16)}$ and increases nutritional intake, mobility, and cognition. ${ }^{(17,18,19)}$ Relationship-building among LC students and residents is significant as it provides students with the opportunity to see the 'person with abilities' behind the 'resident with ailments', allowing for a different perception of seniors. Ensuring that these relationships are valued contributes to the career longevity of an NA. ${ }^{(20)}$

Most importantly, the LC provides several benefits to the group of $\mathrm{NH}$ residents and families. Immediate benefits include additional opportunities for engagement and interaction. Previous research emphasized the important value of personal connections between students and seniors as this influences students' career decisions. ${ }^{(21)}$ Long-term advantages of the LC approach include a larger and better prepared workforce to care for seniors.

The collaborative between Conestoga College and Schlegel Villages has successfully sustained LCs since 2009. Detailed findings of a mixed methods evaluation study will be published soon, but overall findings have been promising. Students involved in the LC demonstrated higher knowledge, behaviours, and skills in gerontology upon graduation in comparison to their counterparts at other campuses, and students had higher job satisfaction and work adaption skills once employed, as compared to other recent graduates. The intentional involvement of $\mathrm{NH}$ residents and staff in teaching was successful, and hiring statistics indicated that the majority of LC graduates found employment in seniors care.

As the LC is an on-going development, some limitations should be discussed. It is important to point out that an evaluative study yet to be published was conducted with participants in one LC and therefore, generalization of the findings may be limited. Further research studies need to include more rigorous evaluations of the LC including resident outcomes, quality of care measurements, and sustainability of the LC. A second limitation is related to the small scope of the current LCs. To the best of our knowledge, there is only one college that is engaged with two NHs in this endeavour. This small sample limits the opportunities for broader discussions to optimize the current LC concepts. To address this concerns and encourage others to engage in this type of innovative learning, we have developed an Implementation Guide for the LC. This guide is available at the Schlegel-UW Research Institute for Aging website. ${ }^{(13)}$

\section{CONCLUSION}

Learning takes place in supportive environments, guided by knowledgeable and inspirational role models. Innovative learning approaches, such as the LC, have great potential to attract students to gerontology, and influence career choices by offering learning environments where students gain real-life experience. Student learning embedded in health-care settings offers students a scope of education beyond that of any typical classroom setting and the ability to engage in practice while learning. With such contributions, NH environments can become innovative hubs for learning, and the input from $\mathrm{NH}$ teams, residents, and LC students all contribute to integrated learning, a better prepared workforce, and ultimately, better care for seniors. LC graduates have the capability and motivation to provide quality care and services for seniors in care settings, and are well-positioned to become future leaders in their field.

\section{ACKNOWLEDGEMENTS}

The authors thank the leadership and teams at Conestoga College, Schlegel Villages, and Schlegel-University of Waterloo Research Institute for Aging (RIA) for their collaboration which permitted the development of the Living Classroom. We would like to acknowledge Ms. Marlene Raasok who held the Executive Dean position of the School for Health and Life Sciences \& Community Services at Conestoga College Institute of Technology \& Advanced Learning at the time of manuscript writing. Financial report was received from the Canadian Institute of Health Research (CIHR) - Catalyst grant (\#126213), Conestoga College, Schlegel Villages, the 
Schlegel Centre for Learning, Research and Innovation in Long-term care (Ministry of Health and Long-Term Care), and the Schlegel-University of Waterloo Research Institute for Aging (RIA). VB receives salary support from the Natural Sciences and Engineering Research Council of Canada (NSERC), administered by CIHR.

\section{CONFLICT OF INTEREST DISCLOSURES}

The authors declare that no conflicts of interest exist.

\section{REFERENCES}

1. Powell JL. The power of global aging. Ageing Int. 2010; 35(1):1-14. Accessed 2016 Feb 24. Available from http://link. springer.com/article/10.1007\%2Fs12126-010-9051-6

2. Statistics Canada. Canada's population estimates: age and sex [Internet]. Ottawa: Government of Canada; 2015. (cited 2016 Nov 1). Available from: http://www.statcan.gc.ca/daily-quotidien /150929/dq150929b-eng.htm

3. CIHI. Health care in Canada, 2011: a focus on seniors and aging [Internet slide show]. Ottawa: Canadian Institute for Health Information; 2011 (cited 2016 Feb 18). 162 screens. Available from: https://secure.cihi.ca/free products/HCIC 2011 seniors report_en.pdf

4. McCleary L, McGilton K, Boscart V, et al. A. Improving gerontology content in baccalaureate nursing education through knowledge transfer to nurse educators. Nurs Leadership. 2009; 22(3):33-46. Accessed 2016 Feb 18. Available from http:// www.abeoudshoorn.com/publications/mccleary2009.pdf

5. Gilje F, Lacey L, \& Moore C. Gerontology and geriatric issues and trends in U.S. nursing programs: a national survey. J Prof Nurs. 2007;23(1):21-29. Accessed 2016 Feb 17. Available from http://primo.tug-libraries.on.ca/primo_library/libweb/ action/dlDisplay.do?docId=TN_sciversesciencedirect_elsevier S8755-7223(06)00191-8\&loc=adaptor,primo_central multiple_fe\&institution=WATERLOO\&vid=WATERLOO\&on Campus $=$ false \&ind $\mathrm{x}=1 \&$ bulkSize $=2 \&$ dym $=$ true \&highlight $=$ tr ue\&lang $=$ eng $\&$ group $=$ GUEST\&query $=$ any,contains, gaps $\% 20$ in $\% 20$ gerontology $\% 20$ education

6. Deschodt M, Dierckx de Casterlé B, \& Milisen K. Gerontological care in nursing education programmes. J Adv Nurs. 2010;66(1):139-148. Accessed 2016 Feb 17. Available from: http://onlinelibrary.wiley.com/doi/10.1111/j.1365-2648. 2009.05160.x/full

7. Baumbusch JL. \& Andrusyszyn M. Gerontological content in Canadian baccalaureate nursing programs: cause for concern? Can J Nurs Res. 2002;34(1):119-129. Accessed 2016 Feb 17. Available from: http://search.ebscohost.com/login.aspx? direct $=$ true $\& \mathrm{db}=\operatorname{cin} 20 \& \mathrm{AN}=2002090481 \&$ site $=$ eds-live

8. COU. Integrating clinical education into Ontario's changing health care system. (COU No. 881). Toronto: Council of Ontario Universities; 2013 (cited 2016 Feb 18). Available from: http:// cou.on.ca/wp-content/uploads/2015/05/Integrating-Clinical -Education-into-the-Changing-Healthcare-System.pdf
9. Campbell SL \& Jeffers BR. The sister model: a framework for academic and service partnerships in nursing home settings. J Gerontol Nurs. 2008;34(9):18-24. Accessed 2016 Feb 17. Available from: http://search.ebscohost.com/login.aspx?direct $=$ true $\& \mathrm{db}=\mathrm{c} 8 \mathrm{~h} \& \mathrm{AN}=2010019923 \&$ site $=$ eds-live

10. Wieland D, Rubenstein LZ, Ouslander JG, et al. Organizing an academic nursing home: impacts on institutionalized elderly. JAMA. 1986;255(19):2622-27. Accessed 2016 Feb 18. Available from http://search.ebscohost.com/login.aspx?direct $=$ true $\& \mathrm{db}=$ cmedm $\& \mathrm{AN}=3701976 \&$ site $=$ eds-live

11. Mezey MD, Mitty EL, \& Bottrell M. The teaching nursing home program: enduring educational outcomes. Nurs Outlook. 1997;45(3):133-140. Accessed 2016 Feb 17. Available from: http:// www.nursingoutlook.org/article/S0029-6554(97)90044-0/abstract

12. Mezey $M$ \& Lynaugh J. Teaching nursing home program: a lesson in quality. Geriatr Nurs. 1991; 12(2);76-77. Accessed 2016 Feb 17. Available from http://search.ebscohost.com/login. aspx direct $=$ true $\& \mathrm{db}=$ cmedm $\& A N=1667130 \&$ site $=$ eds-live

13. Research Institute for Aging. Living classroom implementation guide. Waterloo, ON: Research Institute for Aging; 2016. Retrieved from: http://www.the-ria.ca/livingclassroomguide/

14. Kopera-Frye K, Mahaffy J, \& Svare GM. The map to curriculum alignment and improvement. CELT. 2008;1: 8-14. Accessed 2016 Feb 18. Available from http://celt.uwindsor.ca/ojs/leddy/ index.php/CELT/article/view/3171/2543

15. Gilhooly $M$, Hanlon $P$, Mowat $H$, et al. Successful ageing in an area of deprivation: Part 1- A qualitative exploration of the role of life experiences in good health in old age. Public Health. 2007; 121 (11):807-813. Accessed 2016 Feb 17. Available from http://ac.elscdn.com/S0033350607000972/1-s2.0-S0033350607000972mainpdf?_tid=87beabc6-d5a3-11e5-8889-00000aacb35d\& acdnat $=1455733583 \quad 3 \mathrm{fc} 713 \mathrm{a} 747 \mathrm{aad} 2727237 \mathrm{fd} 8 \mathrm{ac} 84 \mathrm{a} 8 \mathrm{~b} 8 \mathrm{~d}$

16. Logsdon RG, McCurry SM, \& Teri L. Evidence-based interventions to improve quality of life for individuals with dementia. Alzheim Care Today. 2007;8(4):309-318. Accessed 2016 Feb 17. Available from http://www.ncbi.nlm.nih.gov/pmc/articles/ PMC2585781/

17. Manthorpe J \& Watson R. Poorly served? Eating and dementia. J Adv Nurs. 2003;41(2):162-69. Accessed 2016 Feb 17. Available from http://onlinelibrary.wiley.com/doi/10.1046/j.13652648.2003.02520.x/full

18. Blankevoort CG, van Huevelen MJG, Boersma F, et al. Review of effects of physical activity on strength, balance, mobility and ADL performance in elderly subjects with dementia. Dement Geriatr Cogn Disord. 2010;30(5):392-402. Accessed 2016 Feb 17. Available from http://www.karger.com/Article/Pdf/321357

19. Kramer AF \& Erickson KI. Effects of physical activity on cognition, well-being, and brain: human interventions. Alzheimers Dement. 2007; 3(2 Suppl):S45-S51. Accessed 2016 Feb 17. Available from http://www.sciencedirect.com/science/article/ pii/S1552526007000040

20. McCormack B. A conceptual framework for person-centred practice with older people. Int J Nurs Pract. 2003;9(3):202-209. Accessed 2016 Feb 17. Available from http://www.ncbi.nlm. nih.gov/pubmed/12801252 
21. Chung G. Understanding nursing home worker conceptualizations about good care. Gerontologist. 2013;53(2):246-254. Accessed 2016 Feb 17. Available from http://www.ncbi.nlm. nih.gov/pubmed/?term=Understanding+nursing+home+worker + conceptualizations + about + good + care
Correspondence to: Veronique M. Boscart, RN, PhD, Conestoga Applied Research and Innovation (CARI), Conestoga College Institute of Technology \& Advanced Learning, 299 Doon Valley Drive, Kitchener, ON, N2G 4M4, Canada

E-mail: Veronique.Boscart@conestogac.on.ca 